\title{
Article
}

Subscriber access provided by Heriot-Watt | University Library

\section{Polymer-Supported Photosensitizers for Oxidative Organic Transformations in Flow and Under Visible Light Irradiation}

John M Tobin, Timothy J. D. McCabe, Andrew Prentice, Sarah Holzer, Gareth O. Lloyd, Martin J. Paterson, Valeria Arrighi, Peter A.G. Cormack, and Filipe Vilela

ACS Catal., Just Accepted Manuscript • Publication Date (Web): 02 Jun 2017

Downloaded from http://pubs.acs.org on June 5, 2017

\section{Just Accepted}

"Just Accepted" manuscripts have been peer-reviewed and accepted for publication. They are posted online prior to technical editing, formatting for publication and author proofing. The American Chemical Society provides "Just Accepted" as a free service to the research community to expedite the dissemination of scientific material as soon as possible after acceptance. "Just Accepted" manuscripts appear in full in PDF format accompanied by an HTML abstract. "Just Accepted" manuscripts have been fully peer reviewed, but should not be considered the official version of record. They are accessible to all readers and citable by the Digital Object Identifier (DOI®). "Just Accepted" is an optional service offered to authors. Therefore, the "Just Accepted" Web site may not include all articles that will be published in the journal. After a manuscript is technically edited and formatted, it will be removed from the "Just Accepted" Web site and published as an ASAP article. Note that technical editing may introduce minor changes to the manuscript text and/or graphics which could affect content, and all legal disclaimers and ethical guidelines that apply to the journal pertain. ACS cannot be held responsible for errors or consequences arising from the use of information contained in these "Just Accepted" manuscripts. 


\title{
Polymer-Supported Photosensitizers for Oxidative
}

\section{Organic Transformations in Flow and Under Visible}

\section{Light Irradiation}

\author{
John M. Tobin,,$^{\dagger}$ Timothy J. D. McCabe, ${ }^{\dagger}$ Andrew W. Prentice, ${ }^{\dagger}$ Sarah Holzer, ${ }^{\dagger}$ Gareth O. \\ Lloyd,,$^{\dagger}$ Martin J. Paterson, ${ }^{\dagger}$ Valeria Arrighi, ${ }^{\dagger}$ Peter A. G. Cormack ${ }^{*+}$ and Filipe Vilela ${ }^{* \dagger}$ \\ †School of Engineering and Physical Sciences, Heriot-Watt University, Edinburgh, EH14 4AS, \\ Scotland, United Kingdom. \\ *WestCHEM, Department of Pure and Applied Chemistry, University of Strathclyde, Thomas \\ Graham Building, 295 Cathedral Street, Glasgow, G1 1XL, Scotland, United Kingdom.
}

\begin{abstract}
A 2,1,3-benzothiadiazole (BTZ)-based vinyl crosslinker was synthesized and copolymerized with large excesses of styrene using free radical polymerization to deliver heterogeneous triplet photosensitizers in three distinct physical formats: gels, beads and monoliths. These photosensitizers were employed for the production of singlet oxygen $\left({ }^{1} \mathrm{O}_{2}\right)$ and for the aerobic hydroxylation of aryl boronic acids via superoxide radical anion $\left(\mathrm{O}_{2}{ }^{{ }^{-}}\right)$whereby the materials demonstrated good chemical and photo stability. BTZ-containing beads and monoliths were exploited as photosensitizers in a commercial flow reactor, and ${ }^{1} \mathrm{O}_{2}$ production
\end{abstract}


was also demonstrated using direct sunlight irradiation, with a conversion rate comparable to the rates achieved when using a $420 \mathrm{~nm}$ LED module as the source of photons.

Keywords: flow chemistry, free-radical polymerization, heterogeneous photosensitizer, reactive oxygen species, singlet oxygen, visible light

\section{INTRODUCTION}

New advances in photocatalytic processes (including new photocatalysts) have been made in recent years, not only in the production of energy dense materials, as shown in the field of Solar Fuels (with extensive studies on achieving water-splitting and $\mathrm{CO}_{2}$ reduction), but also in organic synthesis. $^{1-3}$ These efforts represent a true advancement in realizing more sustainable, economical and environmentally friendly strategies for a wide-range of chemical processes.

One photocatalytic process that has been under scrutiny is the generation of singlet oxygen $\left({ }^{1} \mathrm{O}_{2}\right)$ through well-known photosensitization reactions. ${ }^{4,5}$ These reactions proceed under mild conditions, requiring only a photosensitizer, a light source and molecular oxygen. Organic dyes such as Rose Bengal, Eosin Blue and Methylene Blue, among others, are some of the most commonly used photosensitizers in this process. ${ }^{6-11}$ When irradiated with light of an appropriate wavelength, energy is absorbed by the photosensitizer which is excited electronically to the triplet state through an intersystem crossing mechanism. The excited photosensitizer then transfers this energy to the ground state triplet oxygen $\left({ }^{3} \mathrm{O}_{2}\right)$, resulting in the formation of ${ }^{1} \mathrm{O}_{2}$. The electrophilic and oxidizing properties of ${ }^{1} \mathrm{O}_{2}$ have driven its use as an oxidant and as a synthetic reagent in a wide range of applications, such as organic synthesis, ${ }^{12}$ water and wastewater treatment ${ }^{13}$ and photodynamic therapy for the treatment of cancer. ${ }^{14,15}$ 
Another area of catalysis that has come to prominence recently is that of photoredox reactions for organic synthesis. ${ }^{16}$ Along with the generation of ${ }^{1} \mathrm{O}_{2}$, photooxidations use molecular oxygen in air as a green reagent, resulting in highly desirable reaction conditions. However, the reactive species for these reactions involve a superoxide radical anion $\left(\mathrm{O}_{2}{ }^{--}\right)$, not the aforementioned ${ }^{1} \mathrm{O}_{2}$. Formation of $\mathrm{O}_{2}{ }^{--}$can proceed through a photoredox cycle in the presence of an amine base acting as a sacrificial reductant, a photosensitizer capable of occupying the excited triplet state and molecular oxygen. ${ }^{17}$

While both reactive forms of molecular oxygen are generally well-known and have their uses in a variety of applications, many of the photocatalytic materials used rely largely on transition metal complexes or organic dyes, which can lead to several issues. For example, the use of organometallic photosensitizers, while efficient light harvesters, ${ }^{17-21}$ tend to be expensive, unsustainable and toxic. Some photosensitizers are also prone to photobleaching, i.e., the degradation of the photosensitizer due to prolonged exposure to light and/or reactivity with ${ }^{1} \mathrm{O}_{2}{ }^{7}$ This leads to both a reduction in the efficiency and the lifetime of the photosensitizer. Lastly, most of these materials are employed under homogeneous conditions, leading to a cumbersome separation and recovery process of the photosensitizer from the reaction mixture, and ultimately the desired products, which impedes the reusability of the photosensitizer. ${ }^{22}$ These disadvantages demonstrate the need to develop metal-free and photostable materials that can act as heterogeneous photosensitizers in a variety of reactions and conditions.

In principle, the use of heterogeneous photosensitizers offers advantages in practical applications when compared to their homogeneous counterparts because they are easy to isolate, recover and recycle. Immobilization of commonly used homogeneous photosensitizers, such as organic dyes ${ }^{23,24}$ and transition metal complexes, ${ }^{25}$ in polymer matrices has been shown to 
provide a more photostable material. This is due to the polymer matrix acting as an efficient oxygen diffusion barrier whilst inhibiting photobleaching of the photosensitizer. ${ }^{26}$ However, this immobilization technique may lead to a decrease in the quantum yield of ${ }^{1} \mathrm{O}_{2}$ production and therefore its efficiency as a photosensitizer. ${ }^{7}$ Nevertheless, the added benefits of a heterogeneous photosensitizer (ease of recovery, reusability and avoiding contamination of the product) outweigh the lower efficiency of the photocatalytic material.

Recently, we reported the use of conjugated microporous polymers (CMPs) as heterogeneous triplet photosensitizers. Through visible light irradiation, these 2,1,3-benzothiadiazole (BTZ) ${ }^{27-29}$ or 4,4-difluoro-4-bora-3a,4a-diaza-s-indacene (BODIPY)-rich ${ }^{30} \mathrm{CMPs}$ have been shown to be efficient and reusable materials for the production of ${ }^{1} \mathrm{O}_{2}$. Reports employing heterogeneous photocatalysts for photoredox reactions have begun to emerge, primarily through the use of CMPs. ${ }^{31}$ However, along with this work, CMP synthetic strategies largely involve Pd catalysts, resulting in an expensive material that is likely to be contaminated with residual Pd. Residual metal, even at ppb levels, is problematic as it may interfere with catalytic processes or lead to the toxicity of the reaction medium due to leaching. To circumvent this issue, we have developed a photoactive vinyl monomer, based upon the photoactive repeat unit used within the BTZCMPs, ${ }^{27-29}$ which can be incorporated with ease into polymer matrices via free radical copolymerization. The BTZ-based monomer contains two styrene units, which results in an electronic "push-pull" system, in which the BTZ unit acts as the strong electron acceptor and the styrene as a weak electron donor. Similarly to the CMPs, we expected that this system would allow the monomer, and polymers derived from the monomer, to act as photosensitizers for the production of ${ }^{1} \mathrm{O}_{2}$. 
One of the most widely available monomers for the production of polymers is styrene, with over 25 million tons produced globally every year. ${ }^{32}$ It is a highly versatile monomer used to prepare a variety of polymer architectures including linear, branched and crosslinked polymers. Various formats of polystyrene have been described, such as foams, gels, beads and monolithic structures. $^{33}$

Herein, the synthesis and characterization of a photosensitizing crosslinking monomer and its incorporation into polystyrene structures through free radical copolymerization is presented. A BTZ-based crosslinker was designed with two styrene units (Scheme 1) allowing for its exploitation as a co-monomer together with styrene and/or divinylbenzene (DVB) in traditional, as well as more specialized, free radical polymerization techniques. Using 3-5 mol \% only of the BTZ-based crosslinker, three insoluble forms of photoactive polystyrene were synthesized: gels, micrometer-sized polymer beads and monoliths. Computational models of the monomer and polymer repeat unit were investigated to demonstrate their ability to occupy the triplet excited state and thereby act as triplet photosensitizers. To validate these models, both the monomer and corresponding polymers were evaluated for the production of ${ }^{1} \mathrm{O}_{2}$ in chloroform under both batch and flow conditions under visible light irradiation $(420 \mathrm{~nm})$. Furthermore, the same materials were screened as triplet photosensitizers in aerobic hydroxylation of a series of aryl boronic acids via the formation $\mathrm{O}_{2}{ }^{--}$. These materials have been presented as a proof of concept where both computational and catalytic experiments verify them as efficient and stable triplet photosensitizers.

\section{EXPERIMENTAL}


General polymer synthesis and photocatalytic procedures will be described henceforth. All other information pertaining to specific experimental methods not presented here can be found in the ESI.

Synthesis of 4,7-distyrene-2,1,3-benzothiadiazole (St-BTZ). To a dry, 2-necked flask equipped with a magnetic stirring bar and a reflux condenser was added a mixture of 4,7-dibromo-2,1,3benzothiadiazole (147 mg, $0.5 \mathrm{mmol})$, 4-vinylphenylboronic acid (148 mg, $1 \mathrm{mmol})$, and $\mathrm{Pd}\left(\mathrm{PPh}_{3}\right)_{4}(17 \mathrm{mg}, 0.015 \mathrm{mmol})$. The flask was connected to a Schlenk line, evacuated and backfilled three times with $\mathrm{N}_{2}$. In the flask whilst under $\mathrm{N}_{2}$, dry THF (40 mL) was added to dissolve the solid reagents. An aqueous solution $(5 \mathrm{~mL})$ was then prepared with $\mathrm{K}_{2} \mathrm{CO}_{3}(140 \mathrm{mg}, 1$ mmol), degassed for approximately 10 minutes with $\mathrm{N}_{2}$ and added to the organic solution. The mixture

Scheme 1. Reaction schemes for the synthesis of the monomer (St-BTZ) and gel-type polymers (Gel (M\%)). 


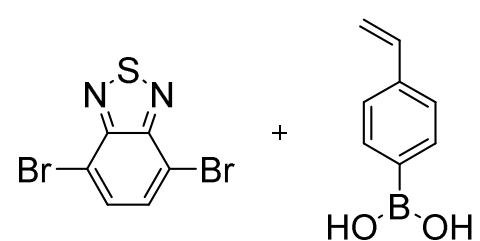

$\mathrm{Pd}\left(\mathrm{PPh}_{3}\right)_{4}, \mathrm{THF}$ $\mathrm{K}_{2} \mathrm{CO}_{3}, \mathrm{H}_{2} \mathrm{O}, 70^{\circ} \mathrm{C}$

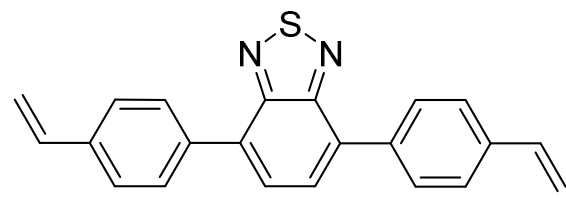

St-BTZ

Styrene, AIBN

Toluene, $80^{\circ} \mathrm{C}$

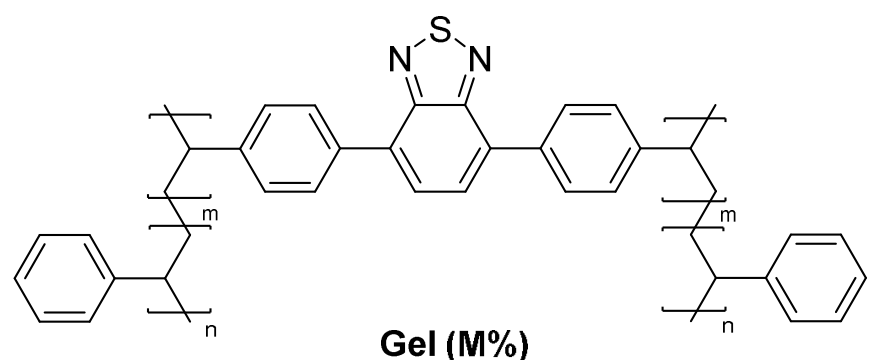

was heated to $70{ }^{\circ} \mathrm{C}$ and left overnight, stirring under a $\mathrm{N}_{2}$ atmosphere. The reaction mixture was allowed to cool to room temperature before adding approximately $40 \mathrm{~mL}$ of water and transferring to a separating funnel with $50 \mathrm{~mL}$ of dichloromethane. The organic layer was washed three times with deionized water and the collected fractions were dried over $\mathrm{Na}_{2} \mathrm{SO}_{4}$. The crude mixture was filtered and purified via silica gel column chromatography with hexane: $\mathrm{CH}_{2} \mathrm{Cl}_{2}$ (4:6) as eluent, yielding a bright yellow colored solid (96 mg, 56\%). ${ }^{1} \mathrm{H}$ NMR $\left(300 \mathrm{MHz}, \mathrm{CDCl}_{3}\right): \delta_{\mathrm{ppm}}=5.36(\mathrm{~d}, 1 \mathrm{H}), 5.89(\mathrm{~d}, 1 \mathrm{H}), 6.85(\mathrm{~m}, 1 \mathrm{H}), 7.63(\mathrm{~d}, 2 \mathrm{H}), 7.83(\mathrm{~s}, 1 \mathrm{H})$, $8.00(\mathrm{~d}, 2 \mathrm{H}) ;{ }^{13} \mathrm{C}$ NMR $\left(100 \mathrm{MHz}, \mathrm{CDCl}_{3}\right): \delta_{\mathrm{ppm}}=114.5,126.5,127.6,129.4,133.0,136.4$, $136.8,137.7,154.1$. 


\section{General synthesis of Styrene-St-BTZ copolymers via free radical polymerization (Gel} (M\%)). Styrene (1 g, $9.6 \mathrm{mmol}$ ) was added to a sample vial with a magnetic stirring bar and dissolved in $5 \mathrm{~mL}$ of degassed toluene. St-BTZ (2, 3 or $5 \mathrm{~mol} \%$ ) and AIBN (16 mg, 0.096 mmol) were added to the solution, allowed to dissolve and degassed with $\mathrm{N}_{2}$ for 10 minutes. The vial was sealed, heated to $60{ }^{\circ} \mathrm{C}$ and left for $16 \mathrm{~h}$ to polymerize while stirring. The solution began as a bright, yellow liquid and changed according to the percentage of St-BTZ added. The reaction was allowed to cool to room temperature and the product was removed from the vial and worked up as follows: (i) Precipitation of product was performed in $100 \mathrm{~mL}$ of $\mathrm{MeOH}$, for soluble copolymers; (ii) Soxhlet extraction was performed for insoluble (crosslinked) copolymers with THF and methanol. All copolymers were dried in vacuo. It is noted that only those polymers containing 3 or more mol \% of the St-BTZ crosslinker produced an insoluble gel. At concentrations lower than $3 \mathrm{~mol} \%$ a soluble polymer was formed. Product recovery: Gel (2\%) 367 mg, $37 \%$; Gel (3\%) 240 mg, $24 \%$; Gel (5\%) 465 mg, $47 \%$.

\section{Synthesis of Styrene-DVB-St-BTZ copolymer via precipitation polymerization (Bead-} BTZ). DVB-55 (221 mg, $1.7 \mathrm{mmol})$, styrene (666 mg, $6.4 \mathrm{mmol})$, St-BTZ (115 mg, $0.3 \mathrm{mmol})$ $(2 \% \mathrm{w} / \mathrm{v}$ total monomer in feed, relative to solvent) and AIBN (41 mg, $0.2 \mathrm{mmol})(2 \mathrm{~mol} \%$ relative to polymerizable double bonds in monomers) were added to acetonitrile $(50 \mathrm{~mL})$ in a Nalgene ${ }^{\circledR}$ bottle $(250 \mathrm{~mL})$. Note that the monomer mole ratio employed for this synthesis was 76:20:4 (styrene:DVB-55:St-BTZ), providing a good comparative analogue to the gels. The bottle contents were ultrasonicated for 15 minutes. After ultrasonication, $\mathrm{N}_{2}$ gas was bubbled through the reaction mixture for 15 minutes in an ice-bath. After degassing, the Nalgene ${ }^{\circledR}$ bottle was sealed under $\mathrm{N}_{2}$ and placed on a low profile roller (Stovall Life Sciences Inc., North Carolina, U.S.A) contained within an incubator (Stuart Scientific, Stone, UK) and rotated slowly 
about its long axis. The temperature inside the incubator was ramped from ambient temperature to $60{ }^{\circ} \mathrm{C}$ over a period of around two hours. The polymerization was allowed to proceed for a further 46 hours to give a milky suspension of polymer particles. The resulting particles were visualized via optical microscopy prior to filtration. The product was filtered by vacuum on a $0.45 \mu \mathrm{m}$ nylon membrane filter. The particles were then washed with solvent to remove any unreacted monomer or initiator $(\sim 100 \mathrm{~mL}$ of acetonitrile followed by $\sim 100 \mathrm{~mL}$ of toluene, methanol and finally acetone) before being dried overnight in vacuo $\left(60 \mathrm{mbar}, 40{ }^{\circ} \mathrm{C}\right)$. Product recovery: $120 \mathrm{mg}, 12 \%$.

\section{Synthesis of Styrene-DVB-St-BTZ copolymer via high internal phase emulsion polymerization (pHIPE-BTZ). Styrene (0.213 g, $2.04 \mathrm{mmol})$, DVB-80 (40 mg, $0.29 \mathrm{mmol})$, St-} BTZ (25 mg, $0.07 \mathrm{mmol})$ and Span $80(0.054 \mathrm{~mL})$ were added to a sample vial and potassium persulfate (18 mg, $0.2 \%$ wt. of water) and sodium chloride (90 mg, $1 \%$ wt. of water) were dissolved in water $(9 \mathrm{~mL})$ in a separate vial. Note that the monomer mole ratio was $85: 12: 3$ (styrene:DVB:St-BTZ), providing a good comparative analogue to the gels. The organic phase was placed in an electric mixer (Polytron PT 1200 E) and the aqueous solution was added slowly. The addition of the aqueous solution was stopped when the emulsion could no longer be mixed properly due to thickening. Remaining liquid was decanted and part of the emulsion was delivered into a transparent glass column ( $7 \mathrm{~mm}$ inner diameter) equipped with porous frit on both ends via syringe. The column was sealed and placed in a $60{ }^{\circ} \mathrm{C}$ oven and left for $24 \mathrm{~h}$. The column containing the synthesized polyHIPE was flushed with $100 \mathrm{~mL}$ of THF, $\mathrm{CH}_{2} \mathrm{Cl}_{2}$ and chloroform. The bright yellow monolith (35 $\mathrm{mm}$ length $\mathrm{x} 7 \mathrm{~mm}$ diameter) was left in the glass column to be used for flow experiments. 
Computational modelling of St-BTZ excited states. Geometry optimization was performed with density functional theory (DFT) using both the CAM-B3LYP and MN12-L functionals, while excited electronic states were computed with time-dependent DFT, utilizing the temporal adiabatic approximation with the same functionals. The 6-311G(d,p) basis set was used for all computations. Analytical Hessian computation was used to confirm optimized stationary geometries as minima. We note only negligible difference between functionals and so discuss the CAM-B3LYP results below (full results for both are given in the supporting information). All computations were performed using Gaussian09. ${ }^{34}$

General procedure for photocatalytic reactions under batch conditions. To a vial containing the required solution $(5-10 \mathrm{~mL}), 5-10 \mathrm{mg}$ of photosensitizing material was added. The mixture was left to stir, whilst open to air, for 10 minutes to allow for proper dissolution or swelling and to aerate the solution. The vial was then placed $7 \mathrm{~cm}$ from a $420 \mathrm{~nm}$ LED lamp and the lamp was switched on. The reaction was monitored via TLC and/or ${ }^{1} \mathrm{H}$ NMR spectroscopy until full consumption of the starting materials was noted, at which time the reaction was stopped and analyzed.

General procedure for photocatalytic reactions in flow employing Bead-BTZ. To a flask containing the required solution (10-15 mL), $5 \mathrm{mg}$ of Bead-BTZ was added. The suspension was first sonicated for 2 minutes to ensure an even dispersion of particles. The mixture was then left to stir, whilst open to air, for 10 minutes to aerate the solution. The dispersion was then pumped through the photochemical reactor (Figure S2) at a flow rate of $1 \mathrm{~mL} \cdot \mathrm{min}^{-1}$. Concurrently, air was pumped through a second pump at the same flow rate, mixing with the dispersion at a Tjunction before entering the photochemical reactor equipped with a $420 \mathrm{~nm}$ LED lamp. The dispersion was cycled for the required time and monitored via TLC and/or ${ }^{1} \mathrm{H}$ NMR 
spectroscopy. Upon consumption of the starting materials, the reaction was stopped and evaluated further.

General procedure for photocatalytic reactions in flow employing pHIPE-BTZ. The pHIPE-BTZ monolith, fitted to a transparent glass column, was allowed to swell in the required solvent for 16 hours. The solution containing the starting materials $(10 \mathrm{~mL})$ was prepared and aerated for 10 minutes open to air. The column was fitted to a light source (Figure S3) and the solution was pumped through the column at $0.5-1.0 \mathrm{~mL} \cdot \mathrm{min}^{-1}$. Concurrently, oxygen was pumped through a second pump at the same flow fate, mixing with the solution at a T-junction before entering the column. The operating pressure was kept at 1.3 bar to ensure a steady stream of both the solution and air. The $10 \mathrm{~mL}$ solution was pumped through the column and monitored via TLC and/or ${ }^{1} \mathrm{H}$ NMR spectroscopy. Upon consumption of the starting materials, the reaction was stopped and evaluated.

\section{RESULTS AND DISCUSSION}

Monomer properties. The UV-Vis absorption spectra for St-BTZ presented as a broad absorption peak in the range 350 to $450 \mathrm{~nm}$ with a maximum absorbance at $400 \mathrm{~nm}$ (Figure S13). This falls within the visible spectrum and is comparable to the absorbance characteristics which we have reported for other BTZ-based photosensitizers. ${ }^{27-29}$ FT-IR, ${ }^{1} \mathrm{H}$ NMR and ${ }^{13} \mathrm{C}$ NMR spectroscopic analysis was fully consistent with the structure of St-BTZ (see ESI). An X-ray crystal structure and the corresponding analysis data can also be found in the ESI.

By virtue of its polymerizable vinyl groups, St-BTZ can be copolymerized with styrene via free radical polymerization. Similarly to DVB, St-BTZ can act as a branching node and consequently as a crosslinker. Employing the crosslinker at an appropriate concentration in a 
copolymerization can result in an insoluble, crosslinked polymer that can then be employed as a heterogeneous photosensitizer.

Synthesis and properties of Gel (M\%). One of the key aims of the work was to minimize the amount of photoactive St-BTZ required whilst ensuring the formation of an insoluble polymer with the desired photosensitizing properties. The selection of styrene as co-monomer as the basis for the heterogeneous photosensitizer was influenced by the stability of polystyrene in the presence of ${ }^{1} \mathrm{O}_{2}{ }^{35}$ and the fact that the photoactive monomer also contains styrene units, which leads to a more compatible copolymerization between the co-monomers.

The formation of these polymers (Gel (3\%) and Gel (5\%)) suggests that there was sufficient crosslinking of the polymer chains to create an insoluble material. The bright yellow color, similar to that of St-BTZ, is also indicative of the integration of the photoactive crosslinker into the polymer network. Most of the solvent used in these reactions was sorbed by the polymers, resulting in bright yellow, swollen gels (Figure 1). A swelling test was performed on dried Gel (3\%), demonstrating an increase of up to seven times its original volume when added to chloroform (Figure S1).

UV-Vis absorption spectra of the materials showed a broad absorption band (350-450 nm) with a maximum at $400 \mathrm{~nm}$ (Figure S13). FT-IR spectroscopic measurements showed peaks corresponding to St-BTZ residues $\left(825\right.$ and $\left.887 \mathrm{~cm}^{-1}\right)$, which were not observed in a polystyrene standard (Figure S12). Note that these signals were relatively weak due to the high ratio of styrene to St-BTZ within the polymer. 


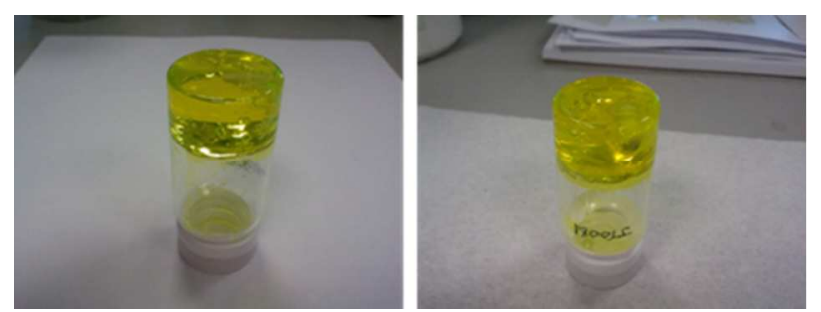

Figure 1. Inverted reaction vials showing Gel (3\%) (left) and Gel (5\%) (right) as insoluble gels swollen in chloroform.

Solid state ${ }^{13} \mathrm{C}$ NMR CP-MAS spectroscopy was also employed to characterize Gel (3\%). Whilst aromatic carbons are present primarily, at $128 \mathrm{ppm}(\mathrm{C}-\mathrm{C})$ and $146 \mathrm{ppm}(\mathrm{C}-\mathrm{N})$, signals in the alkyl region are also identified at 40-45 $\mathrm{ppm}$ and correspond to the saturated aliphatic backbone of the copolymer (Figure S18). These observations suggest that St-BTZ was incorporated successfully into the polystyrene chains via well-established, metal-free, free radical polymerization and that the polymer has the potential to be used a polystyrene-based heterogeneous photosensitizer.

Heterogeneous phase polymerizations (including emulsion, dispersion, precipitation and suspension polymerization $)^{33}$ using the same free radical initiator allow us to demonstrate the versatility of the photoactive crosslinker. This versatility arises from the ability of St-BTZ to be implemented in a variety of free radical polymerization techniques whilst yielding polymers which retain the photoactive properties of the monomer. For the purposes of this study, precipitation polymerization and high internal phase emulsion (HIPE) polymerization were employed, which allows for the synthesis of the polymers in different physical formats derived from the same monomer set: micron-sized beads from precipitation polymerizations and monolithic structures from HIPE polymerizations. 
Precipitation polymerization (Bead-BTZ). As seen in Scheme S2, precipitation polymerization utilizing a free radical initiator was used to synthesize particles in the form of micron-sized beads. In order to ensure the production of an insoluble polymer, divinylbenzene55 (DVB-55) was added as a non-photoactive crosslinker. The incorporation of DVB as a crosslinker also adds rigidity to the structure and good mechanical robustness to the beads. In parallel with the photoactive polymer synthesis, a styrene-DVB copolymer (Bead) was synthesized without any photoactive component for comparison.

UV-Vis spectroscopy showed a maximum absorption at $\sim 420 \mathrm{~nm}$ for Bead-BTZ, which is comparable to the absorbance wavelength of the previously described materials (Figure S15). Bead did not display any absorption in the visible region and produced a white product compared to the bright yellow Bead-BTZ material. An FT-IR spectrum of Bead-BTZ showed the two distinct peaks characteristic of St-BTZ $\left(887\right.$ and $\left.825 \mathrm{~cm}^{-1}\right)$. These peaks were not observed for Bead (Figure S14). Figures $2 \mathrm{a}$ and $2 \mathrm{~b}$ show both bead types together with the corresponding SEM images. These images show the polymer microspheres very clearly, with a bead size range of approximately 1-2 $\mu \mathrm{m}$ for both materials. While Bead resulted in betterdefined particles, Bead-BTZ showed some signs of aggregation and agglomeration. Based on these observations, we concluded that the St-BTZ crosslinker was integrated successfully into the polystyrene beads.

High Internal Phase Emulsion Polymerization (pHIPE-BTZ). The synthesis of pHIPEBTZ was carried out through the free radical polymerization of a high internal phase emulsion (Scheme S3). A UV-Vis spectrum showed a maximum absorption at $\sim 420 \mathrm{~nm}$ for pHIPE-BTZ, which is comparable to the absorption wavelengths of the other synthesized polymers (Figure S15). An FT-IR spectrum showed only weak bands attributable to St-BTZ (887 and $825 \mathrm{~cm}^{-1}$ ) 
a)
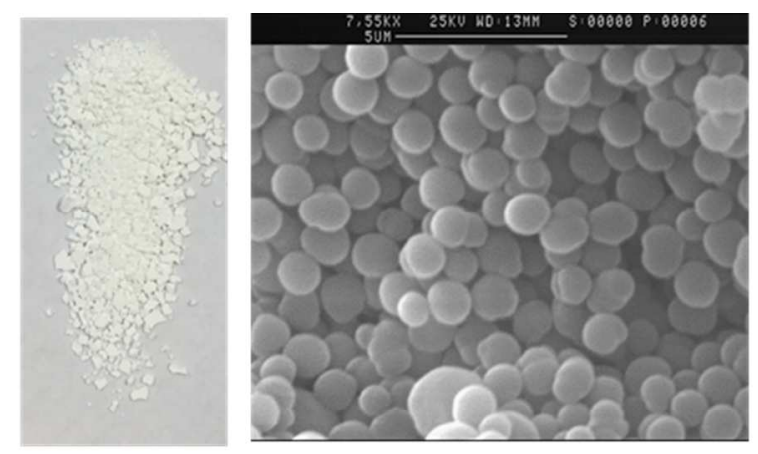

b)
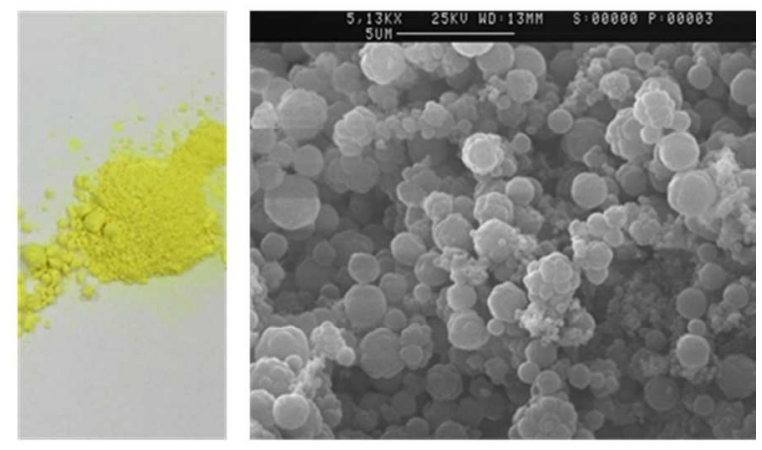

c)
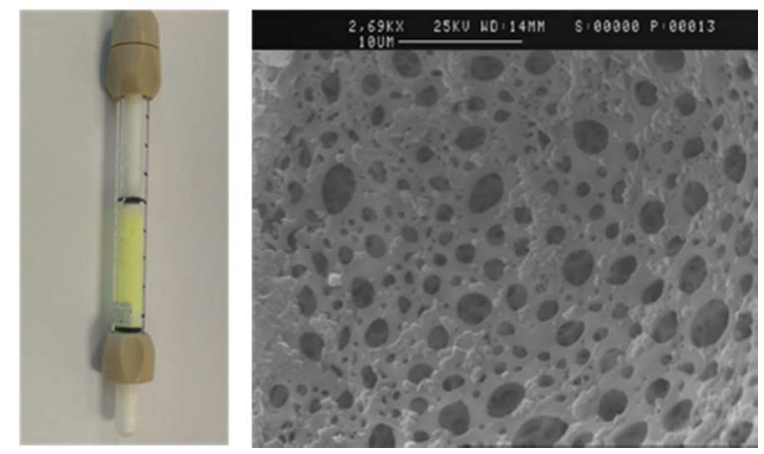

Figure 2. Synthesized copolymers and the corresponding SEM images: a) Bead containing no St-BTZ; b) Bead-BTZ with a yellow color indicating incorporation of St-BTZ; c) pHIPE-BTZ contained within a column as a stationary material for flow applications. 
Given the variety of free-radical polymerization techniques used, the synthesized monomer showed exceptional versatility and stability. By incorporating very limited amounts of the monomer into a polymer matrix, we were able to dilute the amount of photoactive component when compared to more common materials, as previously described. To better understand these materials as potential triplet photosensitizers, computational excited state data were gathered.

St-BTZ excited state computational modelling. Computations have been performed to investigate the lowest singlet and triplet state geometries, and a characterization of vertical electronic excited states, for the St-BTZ monomer and in the absence of vinyl groups as it would appear in the photoactive polymers. For the systems investigated both $\mathrm{C}_{2}$ and $\mathrm{C}_{\mathrm{s}}$ ground state singlet conformers are found (differing in relative sign of dihedral twist for the benzene rings). Each isomer is essentially isoenergetic and results below are for the $C_{2}$ matching the crystal structure (further comparison in ESI). We note generally very good agreement for the $\mathrm{S}_{0}$ geometry with experiment. For both systems the lowest singlet and triplet states are of the same character, being 94\% represented by the HOMO-LUMO orbital transition (Table S1 and S2). This corresponds to charge transfer through the $\pi$-system from the styrene-benzene/isopropylbenzene groups to the BTZ group as shown in Figure 3 below for the monomer and polymer systems. Finally in Table S3 we give the singlet-triplet energy gap computed at the optimized triplet state geometry for both systems. There is around a $0.8 \mathrm{eV}$ triplet stabilization from the vertical geometry for both systems.

Computational verification of the synthesized materials demonstrated their potential to occupy the triplet excited state. These results showed promise and prompted investigations into their catalytic capabilities. For the purposes of this study, we explored their ability to act as triplet photosensitizers in both the production of ${ }^{1} \mathrm{O}_{2}$ and $\mathrm{O}_{2}{ }^{-}$. Furthermore, due to the photoactive 
a)
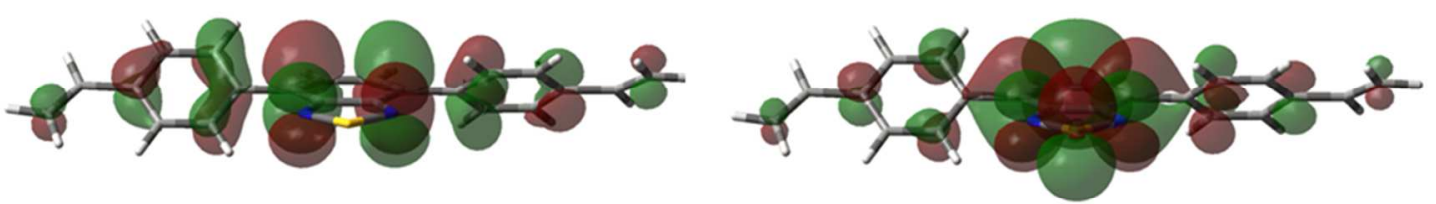

b)
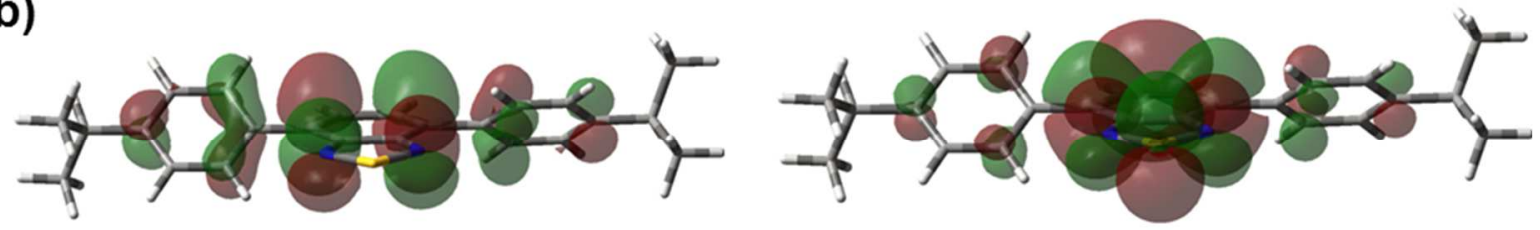

Figure 3. NTO particle-hole representation of a) St-BTZ monomer and b) polymer repeat unit $\mathrm{T}_{1}$ state, computed with TD-CAM-B3LYP/6-311G(d,p).

Homogeneous photosensitization at $420 \mathrm{~nm}$ with St-BTZ. The St-BTZ crosslinker was first examined as a homogeneous triplet photosensitizer in the production of ${ }^{1} \mathrm{O}_{2}$. Scheme 2 shows the Alder-ene reaction, which only proceeds in the presence of ${ }^{1} \mathrm{O}_{2} \cdot{ }^{36}$ The production of ${ }^{1} \mathrm{O}_{2}$ was monitored by observing the conversion of $\alpha$-terpinene into ascaridole via ${ }^{1} \mathrm{H}$ NMR spectroscopy (Figure S20). Full conversion to ascaridole was observed after 90 minutes of irradiation. However, a sudden decrease in the rate of conversion of $\alpha$-terpinene to ascaridole was observed after 45 minutes, causing near full conversion to take upwards of 90 minutes.

Scheme 2. Synthesis of ascaridole from $\alpha$-terpinene via the photosensitization of singlet oxygen under visible light irradiation.

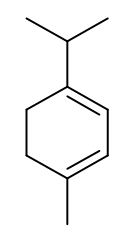

$\alpha$-terpinene

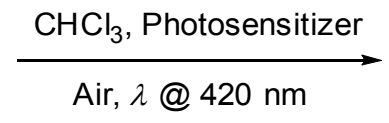
Air, $\lambda @ 420$ nm<smiles>CC(C)C12C=CC(C)(C=C1)OO2</smiles>

Ascaridole 
Samples taken at 15-minute time intervals revealed a dramatic color change (Figure S21), prompting further investigations to determine any potential photo-degradation or oxidative effect on St-BTZ. ${ }^{1}$ H NMR spectroscopic analysis for each sample confirmed the degradation of the photosensitizer, where a clear decrease in the characteristic peaks for the monomer was observed (Figure S20).

Unsurprisingly, interactions between ${ }^{1} \mathrm{O}_{2}$ and vinylarenes can result in the production of monoadducts and endoperoxides. ${ }^{37}$ The reaction potential of the vinyl groups may also have increased due to the electron withdrawing effects of the BTZ component within the monomer. Along with the known reactivity between vinylarenes and ${ }^{1} \mathrm{O}_{2}$, these observations demonstrate the instability of St-BTZ under highly oxidative conditions. Degradation issues aside, we were able to demonstrate the ability of St-BTZ to act as a triplet photosensitizer in the production of ${ }^{1} \mathrm{O}_{2}$ under visible light irradiation. However, we have shown in the past that when incorporating BTZ-based building blocks into CMPs, the stability of the resulting CMP upon production of ${ }^{1} \mathrm{O}_{2}$ is increased when compared to the monomeric version of BTZ. ${ }^{27-29}$ Therefore, and knowing that polystyrene is largely unaffected by ${ }^{1} \mathrm{O}_{2},{ }^{35}$ we postulated that incorporating St-BTZ into a polystyrene matrix would mitigate the photo-degradation or oxidative effect on the polymer.

Heterogeneous photosensitization at $420 \mathrm{~nm}$ with Gel (M\%). A procedure similar to that described above for St-BTZ (Scheme 2) was employed with a reaction time of 60 minutes. Three control experiments were performed first, as follows: (i) in the absence of any photosensitizing material; (ii) using a polystyrene standard as the photosensitizer; (iii) performing the experiment in the dark and in the presence of Gel (3\%). Each of these controls showed no conversion of $\alpha$ terpinene to ascaridole, and therefore that ${ }^{1} \mathrm{O}_{2}$ was not produced under these conditions. 
As seen in Figure 4, both Gel (3\%) and Gel (5\%) showed comparable conversion rates, with close to full conversion achievable after 60 minutes of irradiation. Interestingly, compared to these heterogeneous photosensitization experiments, both homogeneous analogues, St-BTZ and Gel (2\%), gave significantly reduced conversion rates. The reduction in the rate of ${ }^{1} \mathrm{O}_{2}$ production with Gel (2\%) can be explained by a decrease in the level of the photoactive monomer within the material. As the photoactive St-BTZ content decreases, there are fewer accessible sites for an energy transfer to ${ }^{3} \mathrm{O}_{2}$, resulting in reduced production of ${ }^{1} \mathrm{O}_{2}$. The reduced rate of ${ }^{1} \mathrm{O}_{2}$ production with St-BTZ has been explored as explained above. ${ }^{1} \mathrm{H}$ NMR spectra corresponding to each reaction can be found in the ESI.

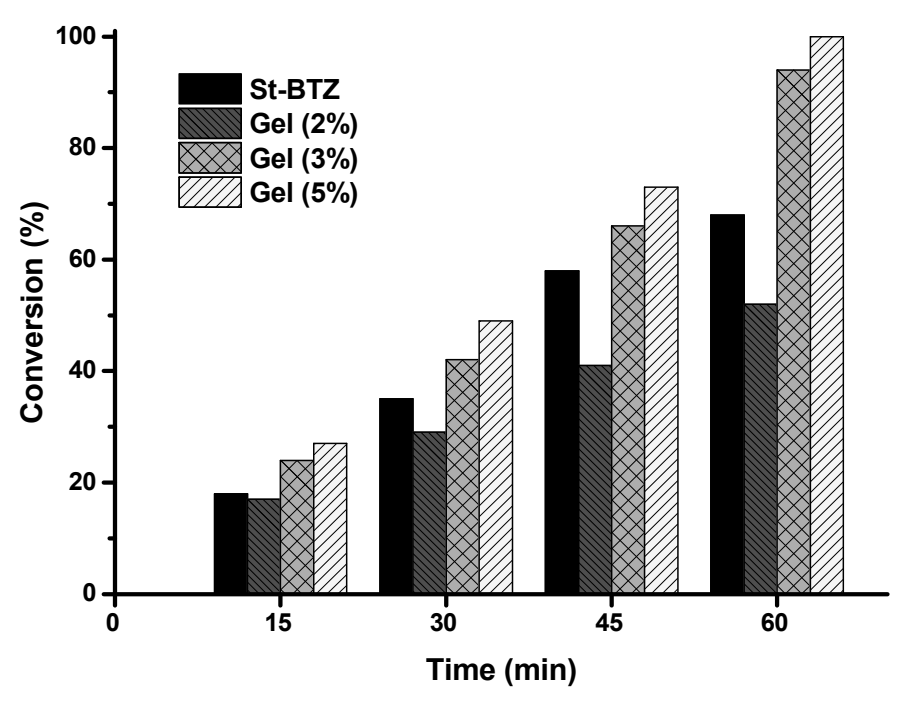

Figure 4. Conversion rates of $\alpha$-terpinene to ascaridole using ${ }^{1} \mathrm{O}_{2}$ produced from the irradiation of St-BTZ, Gel (2\%), Gel (3\%), and Gel (5\%) with a 420 nm LED lamp.

Based on these results, it is clear that the insoluble photoactive gels performed more efficiently than their soluble counterparts. While Gel (5\%) performed slightly better than Gel (3\%), it also required a greater amount of the photoactive monomer. The gels also displayed greater stability 
than St-BTZ; no appreciable color change was observed over the reaction period. Most notably, these results demonstrate the generation of ${ }^{1} \mathrm{O}_{2}$ with a heterogeneous photosensitizer where the main component is polystyrene (95-97\%). This is advantageous and cost-effective since it reduces the amount of St-BTZ required in the copolymer to produce ${ }^{1} \mathrm{O}_{2}$ when compared to other heterogeneous photosensitizers where each repeat unit consist of a BTZ monomer. ${ }^{7,27-29}$ Moreover, self-quenching of the desired triplet state between photocatalytic segments of the polymer can occur if the concentration of the photoactive component is too great. This can greatly hinder the triplet state lifetime, and therefore diminish the effectiveness of the material as a photosensitizer. By reducing the concentration of St-BTZ the self-quenching effect is mitigated greatly without compromising the ability of the polymers to act as photocatalysts. ${ }^{8}$ These concepts are crucial when investigating routes to both reduce the cost and increase the sustainability and effectiveness of photoactive materials.

Application of Bead-BTZ and pHIPE-BTZ in continuous flow. Continuous flow chemistry, as an alternative to standard batch synthesis, offers a flexible route to photochemical reactions, particularly when approaching industrial scales. ${ }^{38}$ Reactions using this method allow for streamlined and continuous production of materials along with the potential for work-up and analysis integration. ${ }^{39}$ This is critically important within industrial processes as it has the potential to both reduce costs and increase productivity. This is especially true for photochemical transformations as the irradiation of light to a solution is more efficient due to the shorter path lengths when compared to traditional batch processes. On larger industrial scales, photon penetration depth and excessive irradiation can be problematic under traditional batch conditions. ${ }^{40}$ Continuous flow addresses these issues through higher surface-to-volume ratios and more efficient heat transfer. This allows for investigations into new synthetic pathways and 
reaction methodologies. ${ }^{41}$ Photochemical reactions have been demonstrated widely using continuous flow methods, even under heterogeneous conditions. ${ }^{29,41}$

While the synthesized gels showed good ${ }^{1} \mathrm{O}_{2}$ production, post-reaction recovery and continuous flow chemistry was impractical. When attempted in flow, the gel particles aggregated and coagulated within the tubing. For this reason, Bead-BTZ and pHIPE-BTZ were designed specifically for two distinct continuous flow applications: (i) The polymer beads present a spherical shape and a low mean particle diameter $(1-2 \mu \mathrm{m})$, which allows for facile dispersion throughout a solvent and low susceptibility to aggregation. These properties allow a dispersion of beads within the reaction mixture to flow through a photochemical reactor with greater ease than amorphous gels. This dispersion can be recirculated through the flow reactor for the time required to complete the reaction; (ii) Unlike the beads, a polyHIPE remains as a stationary photosensitizer within a glass column where the reagents flow through the porous structure whilst being irradiated by an external light source. This results in the continuous production of the target product as long as the polyHIPE remains photoactive.

\section{Heterogeneous photosensitization at $420 \mathrm{~nm}$ with Bead-BTZ and pHIPE-BTZ in a} commercial flow reactor. The freely dispersible beads were investigated both in batch as well as under flow conditions. A batch experiment was set up identical to that of $\mathbf{G e l}(\mathbf{3 \%})$ to compare the production of ${ }^{1} \mathrm{O}_{2} .{ }^{1} \mathrm{H}$ NMR spectroscopy revealed similar conversion rates of $\alpha$ terpinene to ascaridole compared to that of Gel (3\%) (>95\%) (Figure S25). This confirmed the photosensitizing nature of Bead-BTZ and showed no appreciable negative effects due to the added DVB-55.

A continuous flow experiment was then carried out with Bead-BTZ with the experimental set up depicted in Figure 5a. The dispersion was recirculated through the photochemical reactor for 
60 minutes where full conversion to ascaridole was observed via ${ }^{1} \mathrm{H}$ NMR spectroscopy (Figure S26). The production rate for the conversion of $\alpha$-terpinene to ascaridole was found to be 136 mg. $\mathrm{h}^{-1}$. In order to relate this to the amount of photoactive component in the beads, the production rate of ascaridole per $\mathrm{mg}$ of St-BTZ in Bead-BTZ needs to be evaluated. This corresponds to $680 \mathrm{mg} \cdot \mathrm{h}^{-1}$.

pHIPE-BTZ was also employed as a photosensitizer under continuous flow conditions. However, the photochemical reactor was replaced with the column containing the trapped photoactive polyHIPE, as described above (Figure $2 \mathrm{~b}$ ). Due to the reduced contact time with pHIPE-BTZ, some experimental conditions had to be changed: (i) The concentration of the solution was lowered to $0.4 \mathrm{mmol} \alpha$-terpinene in $10 \mathrm{~mL}$ of chloroform; (ii) air was replaced with pure oxygen; (iii) flow rates of both the reaction mixture and oxygen were reduced to 0.5 mL. $\min ^{-1}$ (Figure 5b). Similarly to the gels, pHIPE-BTZ did swell in chloroform. Therefore, the polyHIPE samples were allowed to swell for $16 \mathrm{~h}$ in chloroform prior to the introduction of a reaction mixture. These changes were made to allow for a greater opportunity to yield full conversion to ascaridole after a single pass through the irradiated pHIPE-BTZ column.

a)

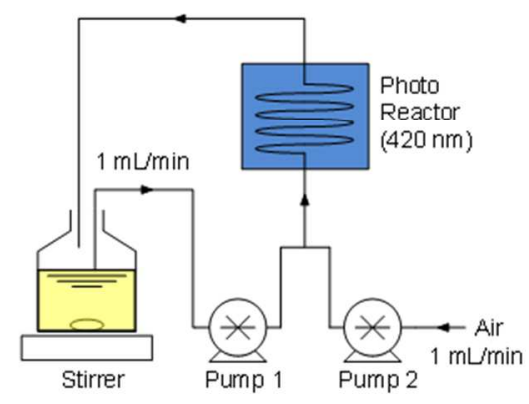

b)

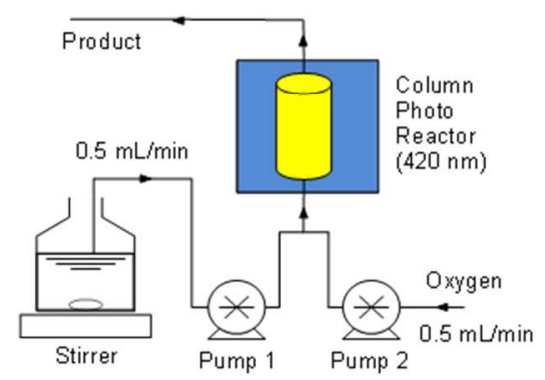

Figure 5. Schematic representation of the experimental set-up using the easy-Photochem flow reactor from Vapourtec Ltd. for a) Bead-BTZ and b) pHIPE-BTZ. 
Under the stated conditions, we observed full conversion of $\alpha$-terpinene to ascaridole after a single pass of the $10 \mathrm{~mL}$ solution of $\alpha$-terpinene $(0.4 \mathrm{mmol}$ in chloroform) (Figure $\mathrm{S} 27)$. To investigate the reusability of pHIPE-BTZ, a continuous stream of the reaction mixture was allowed to flow through the column, taking measurements in $10 \mathrm{~mL}$ increments. After 30 measurements under continuous flow and irradiation, no decrease in the conversion rate was observed (Figure S28). This suggests that the pHIPE-BTZ photosensitizer is stable to both light and ${ }^{1} \mathrm{O}_{2}$ over extended usage periods. Moreover, we note that the same column was employed in the photosensitization of $\mathrm{O}_{2}{ }^{--}$for the aerobic hydroxylation of aryl boronic acids (as described below), demonstrating the superior stability of pHIPE-BTZ and its potential as a reusable photoactive material.

The production rate for the conversion of $\alpha$-terpinene to ascaridole under pHIPE-BTZ conditions was found to be $192 \mathrm{mg} \cdot \mathrm{h}^{-1}$. Thus, both of the continuous flow methods provide specific benefits. The use of Bead-BTZ in flow results in good production rates where larger quantities of material are required. However, the use of pHIPE-BTZ comprises a method in which no intervention is required to obtain the desired product, such as recovery of the polymer from the reaction mixture. These results demonstrate the potential of these stable and reusable materials in heterogeneous photocatalytic applications.

Production of ${ }^{1} \mathrm{O}_{2}$ through photosensitization reactions under sunlight irradiation. To demonstrate further the potential and robustness of these new polymers, a ${ }^{1} \mathrm{O}_{2}$ production experiment was carried out in batch with Gel (3\%) and Bead-BTZ under direct sunlight irradiation as an alternative to the $420 \mathrm{~nm}$ LED modules. All other parameters were kept the same as described above for the batch reactions. The experimental set-up (Figure 6) demonstrates the ease with which the experiment was carried out. Full conversion was observed 
after 90 minutes with Gel (3\%) and after 60 minutes with Bead-BTZ (Figure S29 and S30). Under natural sunlight irradiation and aerobic conditions, along with comparable conversion rates, we were able to validate the robustness of the synthesized photosensitizers. The ability to use sunlight as a free source of energy and air as the source of oxygen demonstrates how two of the main components for this photosensitization reaction greatly increase the potential for low environmental impact applications.

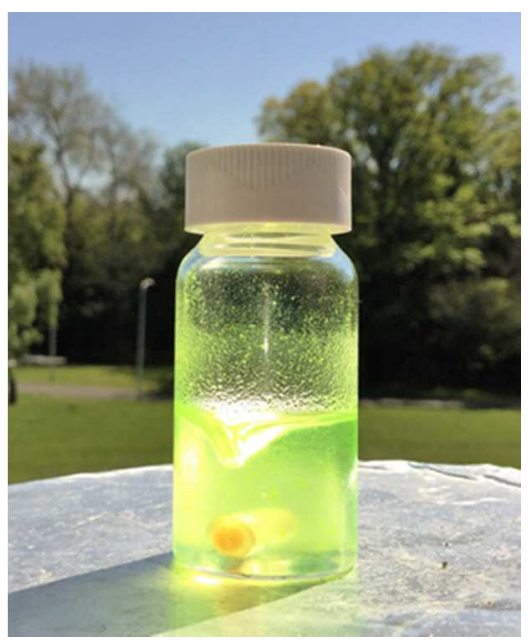

Figure 6. Experimental set-up of the conversion of $\alpha$-terpinene to ascaridole using ${ }^{1} \mathrm{O}_{2}$ produced from direct sunlight irradiation.

Aerobic oxidative hydroxylation of aryl boronic acids. Due to the ability of the described polymers to reach a stable excited triplet state for the generation of ${ }^{1} \mathrm{O}_{2}$, we successfully demonstrated how these materials compare to our previous work. ${ }^{27-29}$ This prompted investigations into the potential of these polymers to act as triplet photosensitizers in other chemical transformations. Materials which can occupy an excited triplet state and subsequently be employed as photocatalysts have been studied in depth and are well known. ${ }^{8}$ Within the applications for which triplet photosensitizers can be employed, we envisaged the use of our photoactive polymers for photoredox reactions. 
One such reaction that has received much attention recently is the aerobic hydroxylation of aryl boronic acids to phenols. Xiao et al. first reported the use of a visible-light photocatalytic strategy to convert aryl boronic acids to phenols using Ru-based complexes and organic dyes. ${ }^{42}$ The large scale synthesis of phenols is of great importance as they have wide applications in the chemical and pharmaceutical industries. However, the photosensitizers employed for this process are largely transition metal-based materials, which can be problematic when trace amounts of these metals remain in the reaction products, as noted previously. More recently, Zhang et al. reported the use of porous carbazole networks as effective visible-light heterogeneous photocatalysts for organic synthesis, including the transformation of aryl boronic acids to phenols. ${ }^{43}$ This demonstrates the growing interest for metal-free, heterogeneous photocatalytic materials. In this context, we implemented the synthesized polymers as heterogeneous photosensitizers for the aerobic oxidative hydroxylation of aryl boronic acids.

A standard model reaction was first performed for the oxidation of phenylboronic acid, demonstrating full conversion via ${ }^{1} \mathrm{H}$ NMR spectroscopic analysis (see ESI) after $24 \mathrm{~h}$. A series of control experiments were then performed, eliminating either light, air, base or the photocatalyst (Table S6). Each of these controls showed that no reaction occurred, indicating the essential requirement of each component for this photocatalytic reaction. St-BTZ was employed as a monomeric comparison to Gel (5\%). While photoactive, the monomer was unable to fully convert phenylboronic acid to phenol within $24 \mathrm{~h}$ and was therefore less efficient. The focus of this model reaction then turned to optimization of the reaction condition. Upon utilization of different solvents $\left(\mathrm{MeOH}, \mathrm{CH}_{3} \mathrm{CN}\right)$ and bases $\left(\mathrm{Et}_{3} \mathrm{~N}, 1,8\right.$-diazabicyclo[5.4.0]undec-7-ene), we discovered that the initial reaction conditions resulted in the swiftest time to full conversion of the boronic acid. 
A scope of reagents for aerobic oxidative hydroxylation of aryl boronic acids is summarized in Table 1. Using the initial optimized reaction conditions, a range of substituted aryl boronic acids were oxidized to the corresponding aryl alcohols until full conversion was demonstrated. A range of electron-neutral, electron-donating and electron-withdrawing substituents were successfully converted to the alcohols. We discovered that among these substrates, those with electrondonating properties (Table 1, Entry 2-3) showed slower conversion rates (40-72 h) compared to phenyl boronic acid. However, those with strong electron-withdrawing substituents (Table 1, Entry 4-6) showed markedly increased conversion rate (18 h) when compared to the other groups. One reagent that did not follow this trend was the ester-substituted phenyl boronic acid (Table 1, Entry 5), showing full conversion after $48 \mathrm{~h}$. Within this scope, we also extended the use of these reaction conditions for the conversion of phenyl boronic pinacol ester, a derivative of phenyl boronic acid, to phenol. To our surprise, full conversion to the phenol product was seen after $15 \mathrm{~h}$, nearly half the time required for the conversion of phenyl boronic acid (Table 1 , Entry 7).

Table 1. Light induced aerobic oxidative hydroxylation of aryl boronic acids. ${ }^{a}$

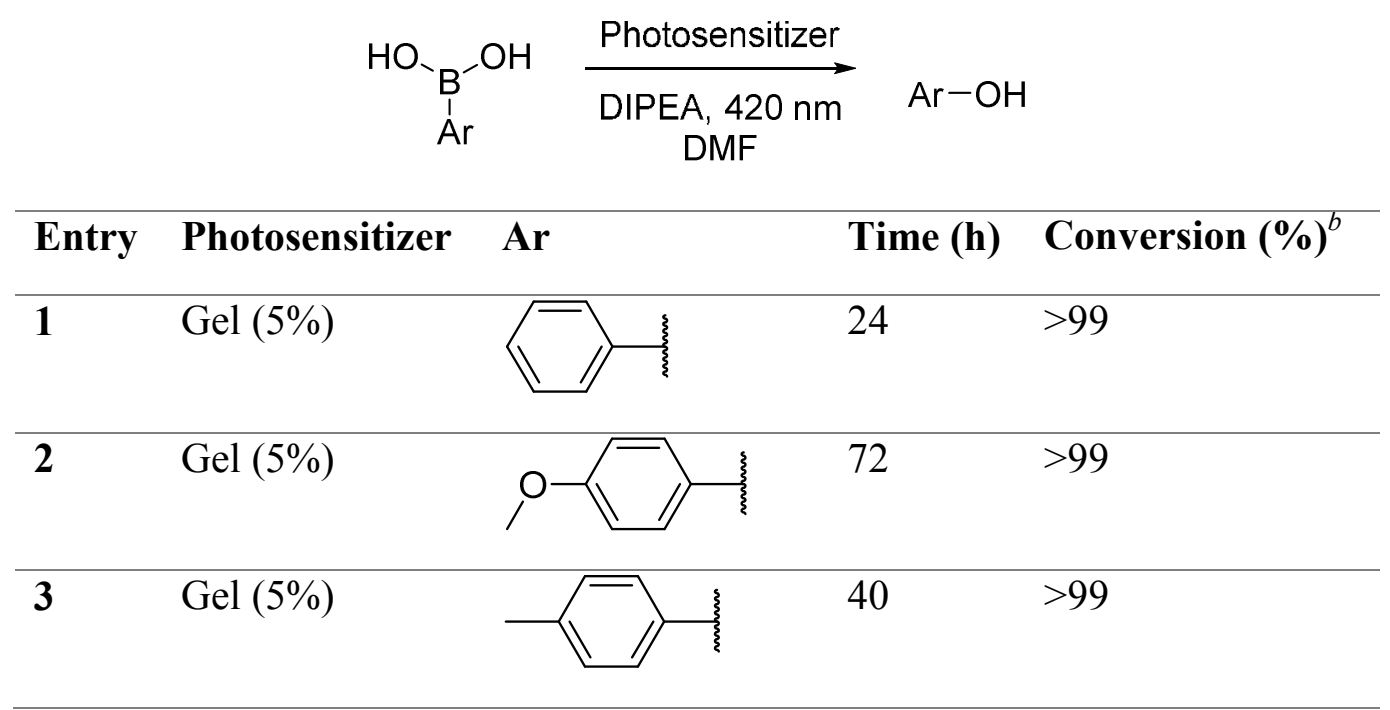




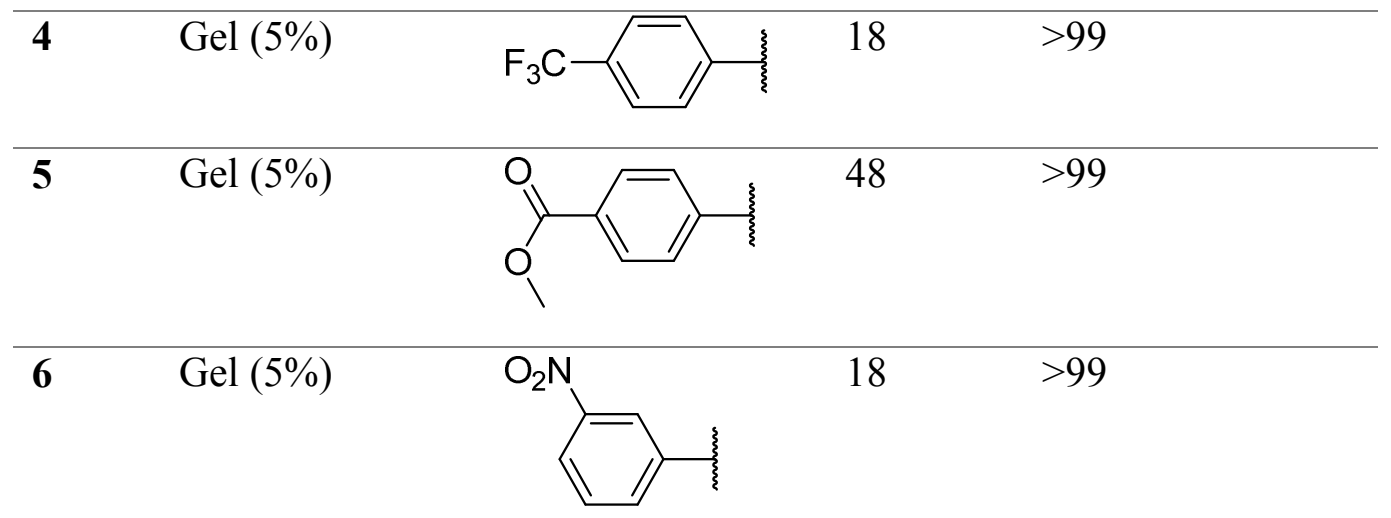

$7 \quad$ Gel $(5 \%)$

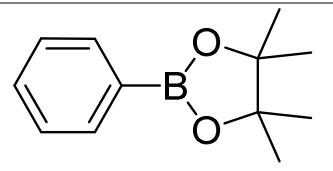

$15>99$

$\mathbf{8}^{c} \quad$ pHIPE-BTZ

\begin{tabular}{lllll}
\hline 9 & $\begin{array}{l}\text { Bead-BTZ } \\
\text { (Batch) }\end{array}$ & 10 & $>99$ \\
\hline $\mathbf{1 0}^{c}$ & $\begin{array}{l}\text { Bead-BTZ } \\
\text { (Flow) }\end{array}$ & 599
\end{tabular}

${ }^{a}$ Reaction conditions: Aryl boronic acid (0.5 mmol/5 mL DMF), Gel (5\%) (5 mg), DIPEA (1.0 mmol), DMF (5 mL), $420 \mathrm{~nm}$ LED irradiation, air, 24 h. ${ }^{b}$ Conversion calculated via ${ }^{1} \mathrm{H}$ NMR spectroscopy. DIPEA $=N, N^{\prime}$-diisopropylethylamine, $\mathrm{DMF}=N, N^{\prime}$-dimethylformamide. ${ }^{c}$ Reaction performed under flow conditions.

Investigations into the mechanism of this reaction have been discussed within the literature and we believe the above reactions proceed in a similar fashion, through the generation of superoxide radicals via an electron transfer mechanism between the triplet photosensitizer and an amine base. $^{42,43}$ By removing the base and subsequent source of superoxide radicals, we have shown that ${ }^{1} \mathrm{O}_{2}$, which we know is generated, has no effect on the aryl boronic acids. However, we have not found any experimental studies within the literature that investigate the generation of ${ }^{1} \mathrm{O}_{2}$ along with $\mathrm{O}_{2}{ }^{--}$in the presence of a base. To test this, $\alpha$-terpinene, which is well known to react only with ${ }^{1} \mathrm{O}_{2},{ }^{36}$ was added to the model reaction. The resultant ${ }^{1} \mathrm{H}$ NMR spectrum (Figure S45) 
showed the presence of both the phenol and ascaridole products, confirming the concomitant production ${ }^{1} \mathrm{O}_{2}$ and $\mathrm{O}_{2}{ }^{--}$in the same reaction. While this may not have any particular benefit regarding synthetic procedures, it is important to recognize as a potential issue when reactants or products are particularly susceptible to an unwanted attack by ${ }^{1} \mathrm{O}_{2}$.

As a further showcase of the synthesized polymers as versatile heterogeneous triplet photosensitizers, we implemented the optimized model reaction under flow conditions, employing both pHIPE-BTZ and Bead-BTZ. The use of the immobilized pHIPE-BTZ resulted in a time of $30 \mathrm{~h}$ to reach full conversion (Table 1, Entry 8). While this is a significantly longer reaction time when compared to the model reaction in batch, we note that the total usage of the photoactive polyHIPE at this point was around $80 \mathrm{~h}$. During this time, the polyHIPE was exposed to direct light irradiation, a variety of solvent and chemical systems and harsh oxidative conditions. The fact that pHIPE-BTZ continued to display good photocatalytic activity after such an extended and extreme use illustrates the robust and nearly indestructible nature of the material. Implementation of Bead-BTZ under both batch and flow conditions was also performed, both resulting in unsurprisingly accelerated conversion rates. Under batch conditions the optimized model reaction required $10 \mathrm{~h}$ to show full conversion to the phenol product, more than halving the time required when employing Gel (5\%) as the photosensitizer. Under flow conditions, the same reaction showed yet another dramatic decrease in reaction time with full conversion occurring after only $5 \mathrm{~h}$. When comparing these results to those of ${ }^{1} \mathrm{O}_{2}$ generation, a large disparity can be seen with regards to the photoactive capabilities of the polymers under different reaction conditions. While the gels and beads both showed comparable reaction rates for ${ }^{1} \mathrm{O}_{2}$ generation, we now see a vast difference in effectiveness for the photoredox reaction. This is most likely due to dissimilar physical characteristics between the two materials. While 
the gels are able to swell greatly, they do not disperse as well as the beads in the same solvent systems. The shape and size of Bead-BTZ aids in this as they form well distributed suspensions, which can create a larger surface area by which triplet state interactions can occur. This idea can also be applied to the decreased reaction time required for continuous flow over batch conditions. A suspension can cause light penetration difficulties, resulting in areas of the reaction mixture receiving lower flux and therefore a decrease in triplet state interactions. By applying the same reaction in flow, we negated this issue as demonstrated by the change in the rate of reaction.

Lastly, due to the enhanced capabilities of Bead-BTZ, we aimed to test both the reusability and productivity of the beads under flow conditions using the same model reaction. We found that beads showed good reusability, with full conversion occurring within the same time as the first run (5 h) after six subsequent uses. Furthermore, and as explained previously, the pHIPEBTZ monolith was used over 30 times for the production of ${ }^{1} \mathrm{O}_{2}$ and then employed in the light induced aerobic oxidative hydroxylation of aryl boronic acids, showing great photostability, stability towards reactive oxygen species, and that it can be reused easily for both of the reactions. The productivity of the material was investigated by determining the maximum concentration that can be implemented where full conversion was still achieved after $5 \mathrm{~h}$. From a starting concentration of $0.1 \mathrm{mmol} . \mathrm{mL}^{-1}$, we successfully increased this to $0.4 \mathrm{mmol} . \mathrm{mL}^{-1}$ before longer reaction times were required. The dramatic reduction in reaction time, along with reusability and productivity studies, further validates the use of continuous flow technology as a tool for enhancing and optimizing reaction protocols even in heterogeneous photocatalysis.

\section{CONCLUSION}


A novel BTZ-based crosslinking monomer was synthesized and successfully copolymerized with styrene to produce three insoluble polystyrene-based photosensitizers in different formats: gels, beads and monoliths. These materials exhibited absorption wavelengths in the visible spectrum $(400-420 \mathrm{~nm})$ and computational data suggested their ability to occupy the triplet excited state. Thereafter, and as a proof of concept, the materials were employed successfully as triplet photosensitizers in the production of ${ }^{1} \mathrm{O}_{2}$ and $\mathrm{O}_{2}{ }^{-{ }^{--}}$under different conditions. Whilst the photoactive monomer demonstrated good photosensitizing properties, it was revealed to be unstable in the presence of ${ }^{1} \mathrm{O}_{2}$. Through its incorporation into a polystyrene matrix, chemical stability was improved dramatically without the loss of its photoactive properties and showed better performance even at low concentrations. While the polymer gels proved to be efficient triplet photosensitizers under batch conditions, they were not suitable for continuous flow chemistry. Both Bead-BTZ and pHIPE-BTZ, designed for use under continuous flow conditions, were shown to be exceptional photosensitizers. The polyHIPE in particular proved to be an extremely stable and reusable photosensitizer, with total usage time exceeding 80 hours.

These materials have been reported as a proof of concept and through computational and experimental methods, the polymer-supported monomer is clearly demonstrated as a robust triplet photosensitizer. Currently, we are investigating the incorporation of other dyes into polymeric systems through versatile free radical polymerization techniques, particularly within water-compatible polymers for water and waste water treatment ${ }^{44}$ and other systems requiring biocompatability. We acknowledge that photophysical studies are necessary to fully understand the triplet excited state of these materials. However, the primary scope of this work aims to describe the design and applications of a new class of materials. Whilst photophysical 
characterization it is not a focus of the current work, it is an aspect we will look to address in the future.

\section{ASSOCIATED CONTENT}

Supporting Information. Figures of commercial flow reactors; ${ }^{1} \mathrm{H}$ and ${ }^{13} \mathrm{C}$ NMR spectroscopic data; Computational data; UV-Visible spectra; FT-IR spectra. This material is available free of charge via the Internet at http://pubs.acs.org.

\section{AUTHOR INFORMATION}

\section{Corresponding Author}

* F. Vilela. Email: f.vilela@,hw.ac.uk

* P. A. G. Cormack. Email: Peter.Cormack@strath.ac.uk

\section{ACKNOWLEDGMENT}

We would like to acknowledge Vapourtec Ltd. for their valuable technical support. F. Vilela would like to thank Heriot-Watt University and The Royal Society for financial support (RG140169). G. O. Lloyd would like to thank Heriot-Watt University and The Royal Society of Edinburgh and Scottish Government for support through a RSE/SG Personal Research Fellowship. M. J. Paterson would like to thank the EPSRC for funding through the platform grant EP/P001459/1.

\section{REFERENCES}

(1) Xing, J.; Fang, W. Q.; Zhao, H. J.; Yang, H. G. Chem. - Asian J. 2012, 7, 642-657. 
(2) Habisreutinger, S. N.; Schmidt-Mende, L.; Stolarczyk, J. K. Angew. Chem., Int. Ed. 2013, $52,7372-7408$.

(3) Xuan, J.; Xiao, W.-J. Angew. Chem., Int. Ed. 2012, 51, 6828-6838.

(4) Kruk, I. Environmental Toxicology and Chemistry of Oxygen Species Handbook of Environmental Chemistry; Springer-Verlag: Berlin, 1998; pp 5-36.

(5) Schweitzer, C.; Schmidt, R. Chem. Rev. 2003, 103, 1685-1757.

(6) Lamberts, J. J. M.; Neckers, D. C. Tetrahedron 1985, 41, 2183-2190.

(7) DeRosa, M. C.; Crutchley, R. J. Coord. Chem. Rev. 2002, 233-234, 351-371.

(8) Zhao, J.; Wu, W.; Sun, J.; Guo, S. Chem. Soc. Rev. 2013, 42, 5323-5351.

(9) Kamkaew, A.; Lim, S. H.; Lee, H. B.; Kiew, L. V.; Chung, L. Y.; Burgess, K. Chem. Soc. Rev. 2013, 42, 77-88.

(10) Wu, W.; Guo, H.; Wu, W.; Ji, S.; Zhao, J. J. Org. Chem. 2011, 76, 7056-7064.

(11) Wu, W.; Zhao, J.; Guo, H.; Sun, J.; Ji, S.; Wang, Z. Chem. - Eur. J. 2012, 18, 1961-1968.

(12) Kopetzki, D.; Levesque, F.; Seeberger, P. H. Chem. - Eur. J. 2013, 19, 5450-5456.

(13) Canonica, S.; Tratnyek, P. G. Environ. Toxicol. Chem. 2003, 22, 1743-1754.

(14) Bonnett, R. Chem. Soc. Rev. 1995, 24, 19-33.

(15) Awuah, S. G.; You, Y. RSC Adv. 2012, 2, 11169-11183.

(16) Romero, N. A.; Nicewicz, D. A. Chem. Rev. 2016, 116, 10075-10166. 
(17) Punniyamurthy, T.; Velusamy, S.; Iqbal, J. Chem. Rev. 2005, 105, 2329-2364.

(18) Connick, W. B.; Gray, H. B. J. Am. Chem. Soc. 1997, 119, 11620-11627.

(19) Abdel-Shafi, A. A.; Beer, P. D.; Mortimer, R. J.; Wilkinson, F. Phys. Chem. Chem. Phys. 2000, 2, 3137-3144.

(20) Abdel-Shafi, A. A.; Beer, P. D.; Mortimer, R. J.; Wilkinson, F. J. Phys. Chem. A 2000, 104, 192-202.

(21) Goethals, A.; Mugadza, T.; Arslanoglu, Y.; Zugle, R.; Antunes, E.; Hulle, S. W.; Nyokong, T.; Clerck, K. J. Appl. Polym. Sci. 2014, 131, 1-7.

(22) Kuznetsova, N. Sensitization of Singlet Oxygen Formation in Aqueous Media. In Photosensitizers in Medicine, Environment, and Security; Nyokong, T. Ahsen, V., Eds.; Springer: Netherlands, 2012; pp 267-314.

(23) Marin, M. L.; Santos-Juanes, L.; Arques, A.; Amat, A. M.; Miranda, M. A. Chem. Rev. 2012, 112, 1710-1750.

(24) Guo, S.; Zhang, H.; Huang, L.; Guo, Z.; Xiong, G.; Zhao, J. Chem. Commun. 2013, 49, 8689-8691.

(25) Gerdes, R.; Bartels, O.; Schneider, G.; Wohrle, D.; Schulz-Ekloff, G. Polym. Adv. Technol. 2001, 12, 152-160.

(26) Carpentier, R.; Leblanc, R. M.; Mimeault, M. Enzyme Microb. Technol. 1987, 9, 489-493.

(27) Zhang, K.; Kopetzki, D.; Seeberger, P. H.; Antonietti, M.; Vilela, F. Angew. Chem., Int. Ed. 2013, 52, 1432-1436. 
(28) Urakami, H.; Zhang, K.; Vilela, F. Chem. Commun. 2013, 49, 2353-2355.

(29) Zhang, K.; Vobecka, Z.; Tauer, K.; Antonietti, M.; Vilela, F. Chem. Commun. 2013, 49, 11158-11160.

(30) Tobin, J. M.; Liu, J.; Hayes, H.; Demleitner, M.; Ellis, D.; Arrighi, V.; Xu, Z.; Vilela, F. Polym. Chem. 2016, 7, 6662-6670.

(31) Wong, Y. L.; Tobin, J. M.; Xu, Z.; Vilela, F. J. Mat. Chem. A 2016, 4, 18677-18686.

(32) Global Styrene Production - Merchant Research and Consulting Ltd. http://mcgroup.co.uk/news/20130830/global-styrene-production-exceeded-264-milliontonnes.html (accessed May 1, 2017).

(33) Cowie, J. M. G.; Arrighi, V. Polymers: Chemistry and Physics of Modern Materials, $3^{\text {rd }}$ Ed.; CRC Press: Florida, 2008; pp 1-28, 57-97.

(34) Gaussian 09, Revision D.01; Gaussian, Inc.: Wallingford, CT, 2009.

(35) Geuskens, G.; David, K. In The Photo-oxidation of Polymers. A Comparison with Low Molecular Weight Compounds, Plenary Lectures Presented at the Seventh Symposium on Photochemistry, Leuven, Belgium, July 24-28, 1978; Reiser, A., Ed.; Elsevier: London, 1979.

(36) Schenck, G. O.; Ziegler, K. Naturwissenschaften 1944, 32, 157.

(37) Rabek, J. F. Photodegradation of Polymers; Springer-Verlag; Berlin, 1996; pp 51-97.

(38) Baumann, M.; Baxendale, I. R. Beilstein J. Org. Chem. 2013, 9, 1613-1619.

(39) Levesque, F.; Seeberger, P. H. Angew. Chem., Int. Ed. 2012, 51, 1706-1709. 
(40) Centi, G.; Perathoner, S. Methods and Tools of Sustainable Industrial Chemistry: Process Intensification. In Sustainable Industrial Chemistry: Principles, Tools and Industrial Examples; Cavani, F., Centi, G., Perathoner, S., Trifiro, F., Eds.; Wiley-VCH Verlag GmbH \& Co. KGaA: Weinheim, Germany, 2009; pp 199-256.

(41) Rubio-Martinez, M.; Batten, M. P.; Polyzos, A.; Carey, K-C.; Mardel, J. I.; Lim, K-S.; Hill, M. R. Sci. Rep. 2014, 4:5443, 1-5.

(42) Zou, Y.-Q.; Chen, J.-R.; Liu, X.-P.; Lu, L.-Q.; Davis, R. L.; Jorgensen, K. A.; Xiao, W.-J. Angew. Chem., Int. Ed. 2012, 51, 784-788.

(43) Luo, J.; Zhang, X.; Zhang, J. ACS Catal. 2015, 5, 2250-2254.

(44) Shen, J.; Steinbach, R.; Tobin, J. M.; Mouro Nakata, M.; Bower, M.; McCoustra, M. R. S.; Bridle, H.; Arrighi, V.; Vilela, F. Appl. Catal., B 2016, 193, 226-233. 
For Table of Contents only.

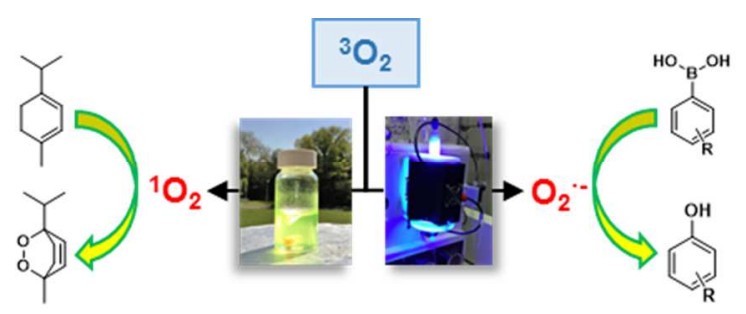

